Review:

\title{
Optical properties of the three polymorphic forms of isotactic polypropylene
}

\author{
Emanuel Nedkov*, Tatyana Dobreva
}

Bulgarian Academy of Sciences, Institute of Polymers, 'akad. G. Bonchev' str., bl.103A, 1113 Sofia, Bulgaria; enedkov@bas.bg

(Received: August 15, 2002; published: October 1, 2002)

\begin{abstract}
A review of the results obtained for the birefringence of different supermolecular structures of three polymorphic forms of polypropylene is presented. All known lamellar super-molecular formations of the monoclinic, hexagonal and triclinic phases are included. The observed morphology varies among negative hedrite and oval structures with axial symmetry, spherulites and typical dendrites. An attempt to explain the observed variety of the various structures' birefringence is made in terms of the mutual orientation of the polarizability ellipsoid and crystallographic axes in the lamellar surfaces.

For the $\alpha$ phase, the orientation of the axes of the polarizability ellipsoid relative to the orientation of the unit cell is determined. The axis $N_{m}$ coincides with the a axis, the fastest growth direction. The hedritic structures, grown by branching homoepitaxy, have negative birefringence. The spherulites differ in intensity and sign of birefringence because of cross-hatching.

For the $\beta$ phase, the crystallographic axis $b$ coincides with $\mathrm{N}_{\mathrm{e}}$, the short axis of the rotational polarizability ellipsoid. The hedritic structures can grow by the screw dislocation mechanism. Spherulites grow by an epitaxy mechanism. Both structures show negative birefringence.

The long axis $\mathrm{N}_{\mathrm{g}}$ of the polarizability ellipsoid of the $\gamma$ phase coincides with the $b$ axis. The short molecules of the $\gamma$ phase are inclined $40^{\circ}$ towards the lamellar surface. The $a$ and $b$ axes do not lie in the lamellar surface. The axes of the polarizability ellipsoid are also inclined towards the lamellar surface. The preferred fast growth direction of the branching lamellae is the $b^{*}$ direction which coincides with $\mathrm{N}_{\mathrm{g}}$, the longest axis in the projection of the optical indicatrix on the lamellar surface. Thus the positive birefringence of the branching lamellae in the mixed $\alpha-\gamma$ spherulites increases more than in the monoclinic positive spherulites.
\end{abstract}

\section{Introduction}

Isotactic polypropylene (IPP) is known to form several crystal modifications - monoclinic $\alpha$ form, hexagonal $\beta$ form and triclinic $\gamma$ form are observed [1]. IPP also exists as a mesophase with paracrystalline structure, named the smectic phase [2]. In all crystal modifications it has the same $3_{1}$ helical conformation, which can have left- and right-handed orientation [3].

The basic single crystal form of the three polymorphic phases is lamellar. The lamellae of the $\alpha$ phase create $\alpha-\alpha$ branches. The branching angle is $80^{\circ}$ towards the surface of the lamellae $[3,4]$. On the other hand, branches do not occur in the $\beta$ modification [4-6]. 
$\gamma-\gamma$ Branches are observed in the $\gamma$ phase. They grow at $40^{\circ}$ towards the parent lamellar surface [7-9]. Lotz at al. also observed $\alpha-\gamma$ branches [7-9].

Several types of supermolecular structures have been described in the literature [5]. Depending on the conditions of formation they are - rhombic and triangle-like hedrites, grown in solution [10], - ovals, obtained by means of slow, isothermal crystallization [5], - and hedrites of the hexagonal phase [5]. Lotz earlier described crystal aggregates of the type 'quadrites', which could be constructed by $\alpha-\alpha$ and $\alpha-\gamma$ branches $[4,7,8]$.

Four types of spherulites are known, depending on the temperature of crystallization and their optical properties [5]. The first two types of spherulites are composed of $\alpha$ lamellae [3]. $\alpha-\alpha$ Branches are often observed in spherulites of $\alpha$ type $[7,8]$, which lead to branching by a type of cross-hatching [3,4,12].

The third and fourth types of spherulites are composed of $\beta$ lamellae. Spherulites of the third type possess a radial structure. They grow at high supercooling. The fourth type of spherulites is banded [3,13].

$\gamma$ Phase spherulites consisting only of $\gamma$ lamellae have not been grown from the melt. The radial and banded $\gamma$ lamellae spherulites can be grown from solutions of IPP irradiated by large doses of $\gamma$-rays [14]. From the melt of IPP, irradiated with different doses, mixed spherulites are formed. That consist of both $\alpha$ and $\gamma$ lamellar branches. At high doses, dendritic spherulites or typical dendrites are formed [15].

There are no data for the orientation of the axes of the optical indicatrix compared to the crystallographic axes of individual lamellae of the three phases. Geil mentions that the hedrites and ovals have a different intensity of birefringence [5]. The birefringence of the spherulites has been better studied. Norton and Keller, and Geil have estimated the value of birefringence of the different kinds of spherulites $[3,5]$.

Birefringence of the two types of $\alpha$ spherulites differs in spite of the fact that they are made by the same monoclinic crystal modification [16]. The first type of $\alpha$ spherulites, which are formed at isothermal or non-isothermal conditions, show weakly positive birefringence. The second type of $\alpha$ spherulites, formed isothermally at low supercooling, shows weakly negative birefringence. At isothermal crystallization, type I spherulites can transform into spherulites of type II. In this transition, the birefringence changes, too $[15,16,17]$. A distinct change in birefringence of spherulites and their extensions, from positive towards negative, is clearly seen during isothermal step-crystallization [16,17]. The opposite transition is possible, too [15]. According to Varga it is due to the $\alpha-\beta$ transition [16]. However, according to Norton and Keller there are only spherulites of the $\alpha$ modification [3].

Spherulites of type III and IV show strongly negative birefringence. Their hexagonal crystal lattice melts at $150^{\circ} \mathrm{C}$. After melting, by keeping at $168^{\circ} \mathrm{C}$, spherulites with weaker birefringence are generated, which is retained during cooling to room temperature. These spherulites originally consist of hexagonal crystals, but after melting they re-crystallize in monoclinic form [5].

Norton and Keller studied the separate types of spherulites and the change in their birefringence depending on the different lamellar structures of the single spherulites $[3,4]$. According to them, type I spherulites are composed of radially and tangentially oriented lamellae, which are due to cross-hatching. However, radially growing lamellae predominate in type II spherulites. Increasing the crystallization temperature or decrease of supercooling is connected with a growth of the radial lamellae, which 
have positive birefringence. Mixed spherulites have areas with radial lamellae, smooth ones, as well as flat regions with zero birefringence [4], and regions with tangential lamellae. The birefringence of both types of lamellae is compensating and, thus, spherulites with zero birefringence sometimes are obtained [3]. Therefore, birefringence changes in intensity and sign in the presence of cross-hatching.

According to Alamo the phenomenon of cross-hatching is significant up to about $136^{\circ} \mathrm{C}$ crystallization temperature; after that it decreases and entirely disappears at about $167^{\circ} \mathrm{C}$. At this temperature they observed the brightest negative spherulites [12]. The lamellar stack is located almost parallel to the plane in which the spherulite grows.

An attempt to explain the great variety of the different polypropylene structures by means of birefringence variation is the aim of the present work. It is based on the mutual orientation of the axes of the polarizability ellipsoid and the crystallographic axes towards the lamellar surface in the different polymorphic modifications.

\section{Results and discussion}

To explain the birefringence of the large number of polylamellar supermolecular structures, one has to compare the orientation of the unit cell axes $(a, b, c)$ with the axes of the polarizability ellipsoid, $\mathrm{N}_{\mathrm{p}}, \mathrm{N}_{\mathrm{m}}$ and $\mathrm{N}_{\mathrm{g}}$. Their mutual orientation has to be defined relative to the lamellar surface $[18,19]$. The obtainment of detached lamellae or large groups of lamellae with the same IPP orientation is strongly restricted [5] or even impossible $[8,12]$.

Below we give suggestions for the mutual orientation of unit cell axes and the axes of the polarizability ellipsoid. The projections of the axes of the unit cells and the disposition of the polarizability ellipsoid towards the lamellar surfaces are compared.

\section{Morphology of the $\alpha$-monoclinic modification in dependence on the temperature of crystallization}

The (001) plane of the monoclinic crystal lattice is presented in Fig. 1. The unit cell axes and the axes of the optical indicatrix are shown in Fig. 2. The longest optical axis $\mathrm{N}_{\mathrm{g}}$ coincides with the $b$ axis, the middle optical axis $\mathrm{N}_{\mathrm{m}}$ coincides with $a$. The $a$ axis is the preferable direction of growth. The shortest $N_{p}$ axis is inclined on the $c$ axis, cf. Fig. 3.

We obtained our information on the orientation of the axes of the polarizability ellipsoid using an empirical rule for monoclinic symmetry, viz. that one of the three axes $N_{p}, N_{m}$ and $N_{g}$ always coincides with the second crystallographic axis $b$. That is why we examined positive and negative $\alpha$ spherulites by light microscopy, observing the interference image in the focal plane in the objective. So named conoscopic observations are made on the parts of supermolecular structures with sizes of about $50 \mu \mathrm{m}$, which have a thickness of about $30 \mu \mathrm{m}$. These part consist of large groups of lamellae with the same orientation. The angle between the optical axes is determined by means of conoscopic observations. We found the plane at obtuse bisectrix, simultaneously in the negative and in the positive monoclinic spherulites. Both monocrystals are positive. Thus we concluded that the second crystallographic axis $b$ coincides with the $\mathrm{N}_{\mathrm{g}}$ axis (Fig. 3). This mutual orientation of the axes of the polarizability ellipsoid and the crystallographic axes towards the lamellar surface does not 
change for the different supermolecular structures - hedrites, quadrites, ovals and spherulites. The birefringence and conoscopic observations of these structures support the models shown in Figs. 2 and 3.

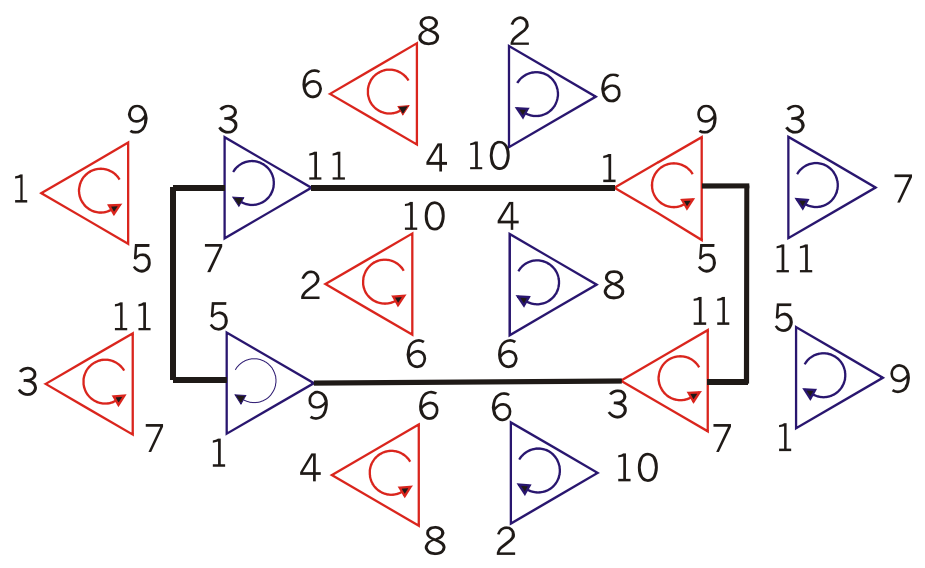

Fig. 1. (from ref. [1]) Projection of the unit cell (bold line) of the $\alpha$ phase of IPP over the plane $\{001\}$. Numbers denote heights of methyl groups above a plane perpendicular to $c$

Fig. 2. Mutual orientation of the crystallographic axes $a, b, c$ of the $\alpha$ crystal lattice towards the axes of the polarizability ellipsoid, $\mathrm{N}_{\mathrm{m}}, \mathrm{N}_{\mathrm{p}}, \mathrm{N}_{\mathrm{g}}$. Sizes and angles of the crystal lattice are noted. Crystallographic axes $a$ and $b$ coincide, respectively, with $\mathrm{N}_{\mathrm{m}}$ and $\mathrm{N}_{\mathrm{g}}$
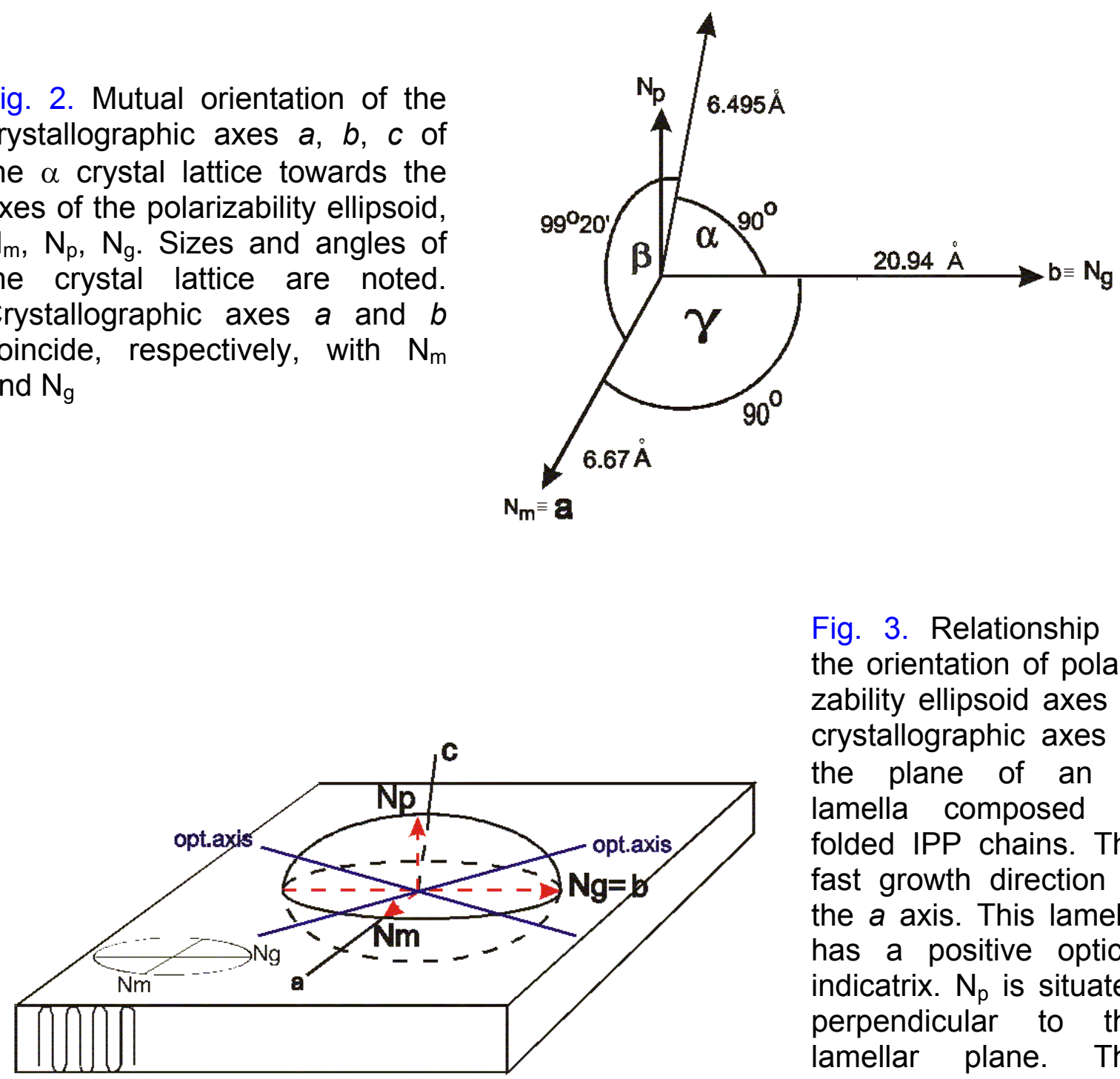

Fig. 3. Relationship of the orientation of polarizability ellipsoid axes to crystallographic axes in the plane of an $\alpha$ lamella composed of folded IPP chains. The fast growth direction is the a axis. This lamella has a positive optical indicatrix. $N_{p}$ is situated perpendicular to the lamellar plane. The other two axes, $\mathrm{N}_{\mathrm{m}}$ and $\mathrm{N}_{\mathrm{g}}$, lie in the plane 
There are known hedritic structures, growing from solution and melt. Lotz's quadrite, which grows from solution, is formed by branching. According to Lotz, wide-angle $80^{\circ}$ $\alpha-\alpha$ branching is unique for synthetic polymers [7]. The formation of branches is not dependent on the molecular weight of IPP, but only on the crystallization conditions. This unique branching on the lamellar surface is due to homoepitaxy. It is typical of the $\alpha$-phase of IPP only [7].

It is known that the hedritic structures grow from the melt at low supercooling, i.e., at temperatures above $140^{\circ} \mathrm{C}[4,5,7,8,20]$. The hedrites have an axial symmetry [4]. Generally, hedritic structures grow with lamellae parallel to the substrate upon which they grow. The basic lamella grows branching epitaxially and multiplies the unit cell's shape [5]. Hedritic structures of other polymers usually grow by screw-dislocation mechanism $[21,22]$. This enhances the multiplication of the unit cell's shape as well as the shape of the basic lamellae. Neither Norton and Keller [3] nor Lotz [7,8] or Binsbergen [4] mentioned growth by a screw-dislocation mechanism for the hedritic structure of monoclinic isotactic polypropylene.

Hedritic structures growing from melt were described by Binsbergen [4]. The primary $\alpha$ lamella, from which the hedritic structure starts to grow, is usually located in parallel to the surface upon which it grows. If we draw its optical indicatrix (Fig. 3) we will see that $N_{g}$ coincides with the $b$ axis, $N_{m}$ coincides with the a axis, and $N_{p}$ is shifted about $10^{\circ}$ from the crystallographic axis $c$. The axis showing the fastest growth is the a axis. This monoclinic lamella should behave like a positive biaxial crystal, because $\mathrm{N}_{\mathrm{g}}$ is the sharp bisectrix in the triaxial polarizability ellipsoid [18]. This basic lamella usually grows branching epitaxially and multiplies the unit cell's shape. If this $\alpha$ lamella is parallel to the substrate upon which it grows, its birefringence will be determined by $\left(\mathrm{N}_{\mathrm{g}}-\mathrm{N}_{\mathrm{m}}\right)$. The $\alpha$ lamellae are capable to create branches, which are almost perpendicular to the basic lamella [8]. Some very small hedritic structures possessing irregular rectangular shape and cross-hatching were described by Binsbergen [figure 11 in ref. 4]. It can be distinctly seen in the same figure that the shape of the very small quadrite is irregular. If very large and thick hedritic monoclinic structures are formed, the shape will be round, and the growth face will be jagged, due to cross-hatching and the storage of a large number of lamellae which are almost parallel to the substrate (Fig. 4).

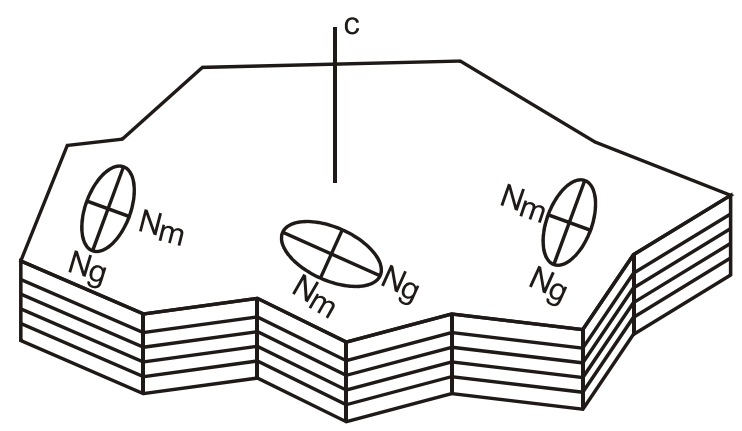

Fig. 4. Hedritic structures built by a lamellae, parallel to each other, with axial symmetry. These structures grow at low supercooling, i.e. at high temperature. It is supposed that cross-hatching is missing [12]. Projections of the polarizability ellipsoid are shown in the plane of this hedritic structure. An irregular growth front may follow the branching of the crystallographic planes. The lamellae in real hedritic structures can deviate considerably from parallel orientation towards the plane over which the primary lamella grows 
Fig. 5 shows a spherulitic-like structure, consisting of $\alpha$ lamellae only, and the jagged face growth is distinct. It can also be seen that the surface of the spherulitic-like hedritic structure is smoother than the surface of its spherulitic growth.

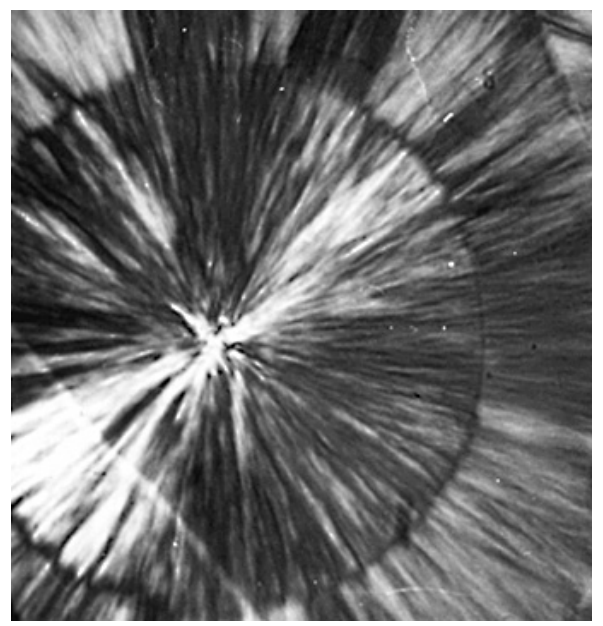

Fig. 5. $\alpha$ Spherulitic-like structure with negative birefringence and positive non-isothermal prolongation. The $\alpha$ spherulitic-like structure has a larger thickness than the spherulitic extensions. IPP, 200x, with gypsum plate; $T_{\mathrm{C}}=140^{\circ} \mathrm{C} ; t=17 \mathrm{~h}$. Thus, our observations, confirmed by some authors, show that if the primary lamella is monoclinic, then all lamellae of the structure are monoclinic, too, i.e., the whole structure is monoclinic $[4,12,15]$. Besides, an $\alpha-\beta$ transition by increasing or decreasing the temperature is not possible, as Varga suggested in an earlier article [17]

It is mentioned that hedritic structures consisting of $\alpha$ lamellae have different birefringence. There are no data for the magnitude of the birefringence, the hedritic structures are only described as lighter or darker [5]. We think that the reason lies in the direction of the stack lamellae growth, which does not always coincide with the crystallographic axis of growth of the basic lamella, i.e., the lamellae turn away and twist. For this reason the type of birefringence strongly changes. The most perfect hedritic structures are formed by lamellae lying parallel to each other and to the substrate over which they grow. In this situation the hedritic structure, as observed by optical microscopy, would have a round growth front and the $\mathrm{N}_{\mathrm{m}}$ axis would be radially oriented and $\mathrm{N}_{\mathrm{g}}$ tangential. This results in negative birefringence for the whole structure (Fig. 4), as observed by many authors [3-6,15,16].

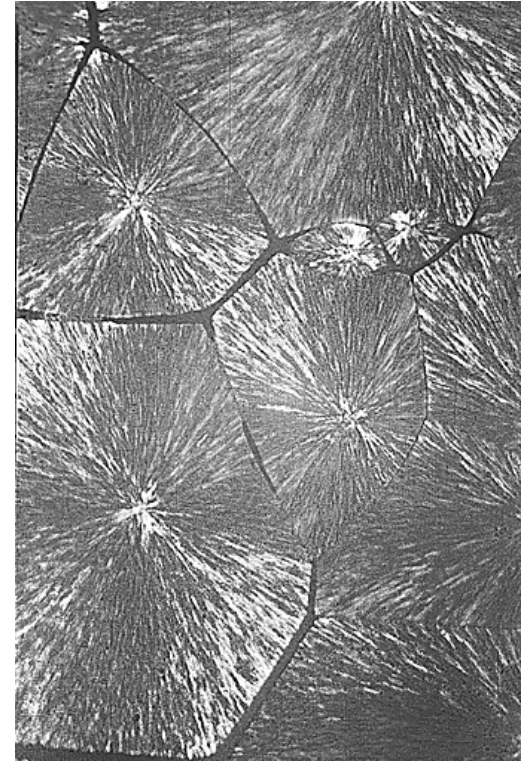

Fig. 6. $\alpha$ Spherulites with weakly positive birefringence, $T_{\mathrm{c}}=130^{\circ} \mathrm{C} ; t=24 \mathrm{~h} ; 200 \mathrm{x}$, with gypsum plate

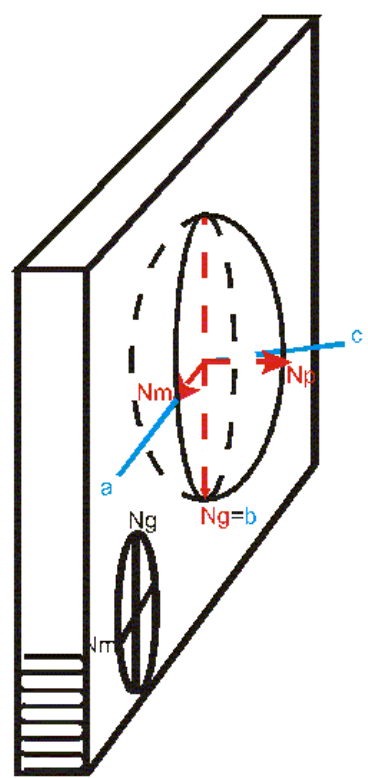

Fig. 7. Radially oriented single lamella in a monoclinic spherulite with weakly positive birefringence 
The spherulitic structures form at high supercooling, i.e. at low temperature, and they grow by a mechanism of secondary nucleation. Small defects on the surface of preceding parent lamellae serve as secondary nuclei. The primary lamellae grow radially towards the spherulitic boundary (Fig. 6). The axis of fastest growth is again the a axis, coinciding with $\mathrm{N}_{\mathrm{m}}$ (Fig. 7). The shortest axis $\mathrm{N}_{\mathrm{p}}$ is tangentially arranged, normal to the spherulitic radius (Fig. 8). It results in a weakly positive birefringence in the $\alpha$ spherulites which grow at high supercooling. Many large alternations in the magnitude of positive birefringence of the spherulites are mentioned in the literature $[3,12]$. This is explained by mutual compensation of the birefringence of the radial and tangential lamellae which grow at an angle of $80^{\circ}$ (Fig. 9 and Fig. 10). With increasing crystallization temperature, the formation of cross-hatching sharply decreases and well formed radially disposed lamella are observed $[4,12]$.

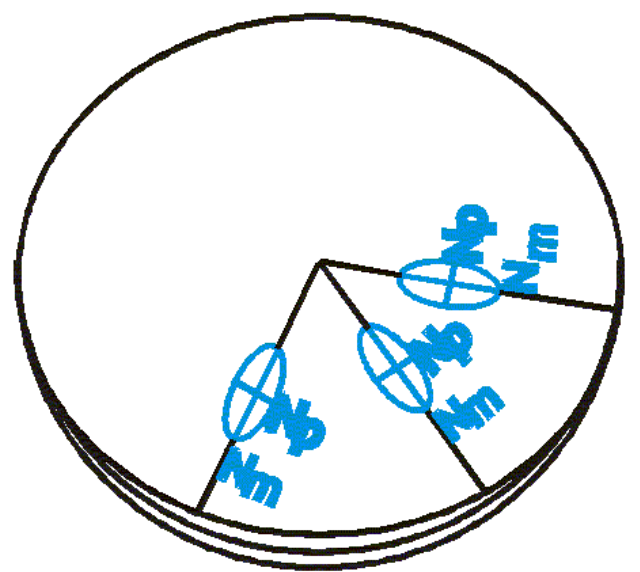

Fig. 8. Positive $\alpha$ spherulite with radially growing lamellae

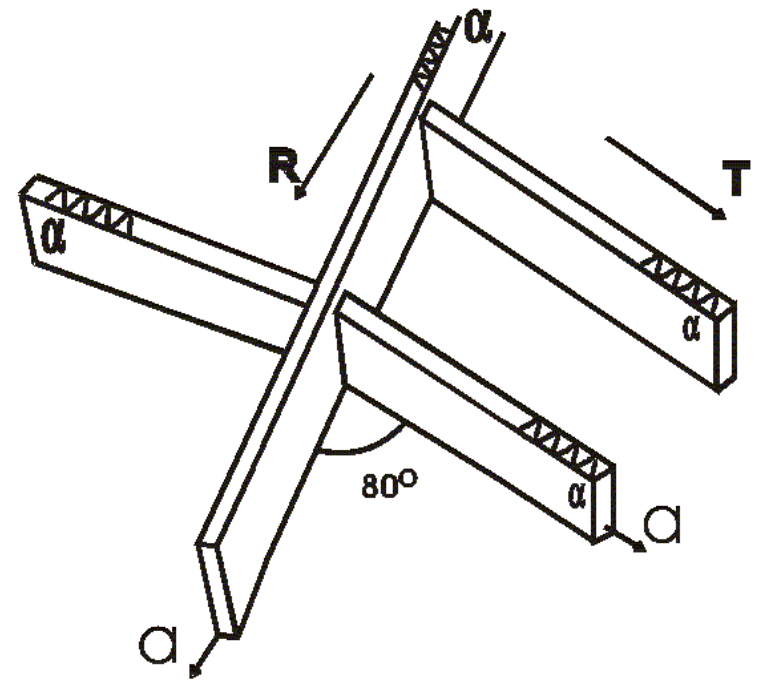

Fig. 9. Cross-hatching or branching. $\mathrm{R}-$ radially growing branches; $\mathrm{T}$ - tangentially growing branches

\section{Morphology of the $\beta$-hexagonal modification in dependence on the temperature of crystallisation}

Ferro et al. have calculated the packing energy of the $\beta$ phase [23]. It is characterized by larger disorder than the $\alpha$ phase. Two structural models have been presented [1] which are characterized by areas of helices with the same chirality, arranged in a pseudo-hexagonal lattice (Fig. 10). A comparative investigation of plastic deformation of one single lamella in $\alpha$ and $\beta$ spherulites [24] shows that the $\beta$ lamellae, under simple shear, are deformed about $25 \%$ more than $\alpha$ lamellae. $\alpha$ Spherulites are probably more stable because of the presence of tangentially grown daughter lamellae.

It is a crystallographic rule that the polarizability ellipsoid is rotational and the crystallographic axes coinciding with the optical axes for the hexagonal phase pass on to the high crystallographic lattices. In this case the optical axis $\mathrm{N}_{\mathrm{o}}$ coincides with the $c$ axis. This coincidence is confirmed by the orthoscopic and conoscopic observations of small parts of the hedritic structures which are depicted by Varga at al. as hexagonites. This mutual orientation of the axes of the polarizability ellipsoid and the crystallographic axes towards the lamellar surface does not change for the different 
supermolecular structures - hedrites, hexagonites, ovals and spherulites. The birefringence and conoscopic observations of these structures support the models shown in Fig. 11 and Fig. 12.

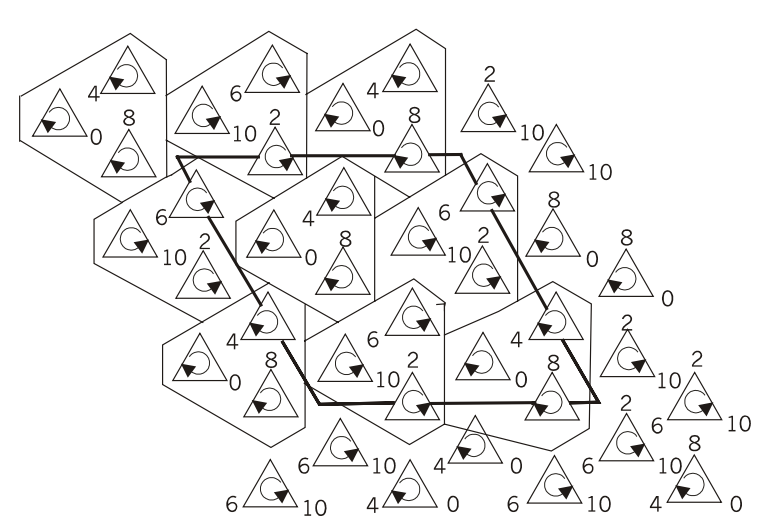

a.

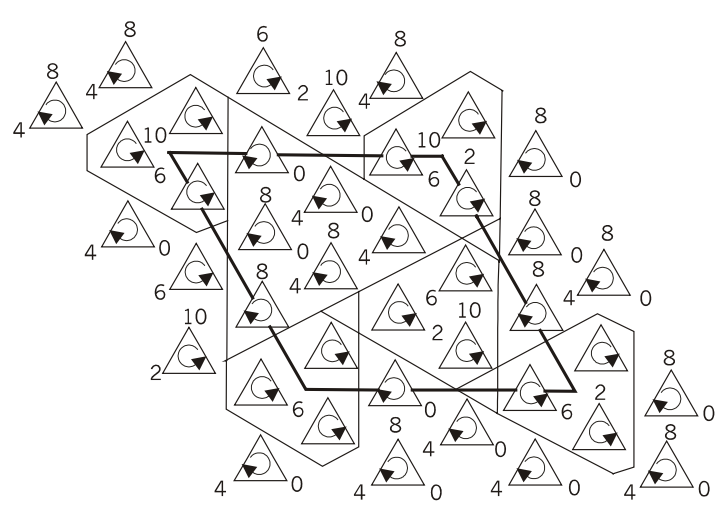

b.

Fig. 10. (from ref. [1]) Possible packing of helices in a 'hexagonal' cell (c projection). Numbers denote heights of methyl groups above a plane perpendicular to $c$. (a) Helices of each hand are arranged in triplets. (b) Helices of one hand are arranged in triplets, and of the other hand in groups of six

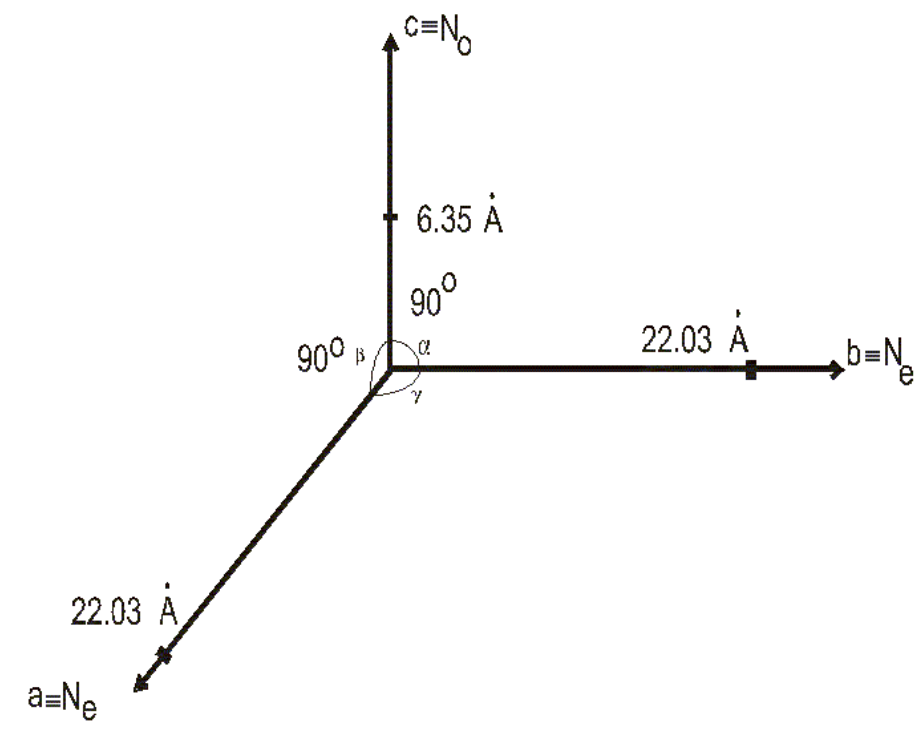

Fig. 11. Axial diagram of orientation of the crystallographic axes relative to the axes of the polarizability ellipsoid, so that a and $b$ coincide with $\mathrm{N}_{\mathrm{e}}$, and $c$ coincides with $\mathrm{N}_{\mathrm{o}}$

Fig. 12. Orientation of the polarizability ellipsoid axes in the plane of a single hexagonal $\beta$ lamella. $\mathrm{N}_{\circ}$ is the optical axis. If the light beam is parallel to $N_{0}$, the lamella will be dark. The a axis is the preferable direction of fast growth of the lamella

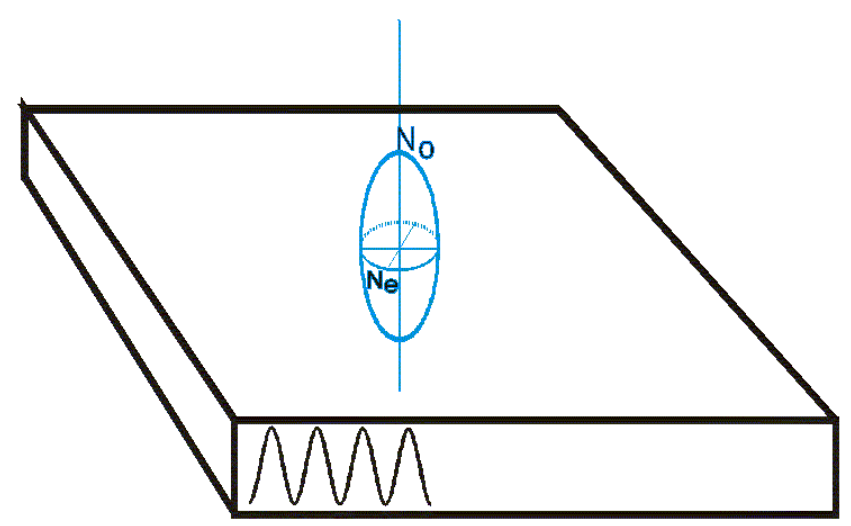


The axial diagram of the $\beta$ hexagonal modification is shown in Fig. 11. The mutual orientation of the crystallographic axes and the axes of the polarizability ellipsoid are as follows: $a$ and $b$ both coincide with $\mathrm{N}_{\mathrm{e}} ; c$ coincides with $\mathrm{N}_{\mathrm{o}}$. The $\mathrm{N}_{\mathrm{e}}$ axis coincides with two crystallographic axes. The preferred direction of fast growth of the hedritic structures is on the a axis [3].

$\beta$ Stacks of lamellae with axial symmetry form in the narrow temperature ranges from 140 to $145^{\circ} \mathrm{C}$ by means of nucleating agents, most often metals [16]. From the melt at high temperature, under applied exterior or existing interior tension, stacks of lamellae could form with axial symmetry $[11,16,26,27,28]$. These lamellae form uniaxial positive crystals. The polarizability ellipsoid is rotationally symmetric (Fig. 12).

The arrangement of lamellae in the hedritic structures can be viewed as the pages in an opened book (ovals) or the pages in a closed book (hedrites of the hexagonite form) $[11,26]$. The hedrites of $\beta$-IPP could form by epitaxial growth $[11,13]$. The hedrites appear like clusters of multilayers of lamellar crystals. The lamellae in ovals (Fig. 13 and Fig. 14) are disposed almost perpendicular to the surface $[11,26]$. The lamellae in the hedrites are disposed parallel to the surface on which they grow. They seem flat $[11,26,28]$. Exactly for $\beta$-IPP, two kinds of lamellae are known; those which are parallel to the surface and those which are tilted from this surface. The first ones are named flat-on. When they grow parallel to the surface, they are dark and their birefringence is very weak (Fig. 12). They have negative birefringence. The birefringence depends on the relative thickness of flat-on lamellae [11]. The so called edge-on lamellae predominate in the ovals $[11,26]$. This type of lamellae can be helically twisted along the $a$ axis which is the preferred fast growth direction $[11,26,28]$.

Fig. 13. Radially growing $\beta$ lamella and its optical indicatrix

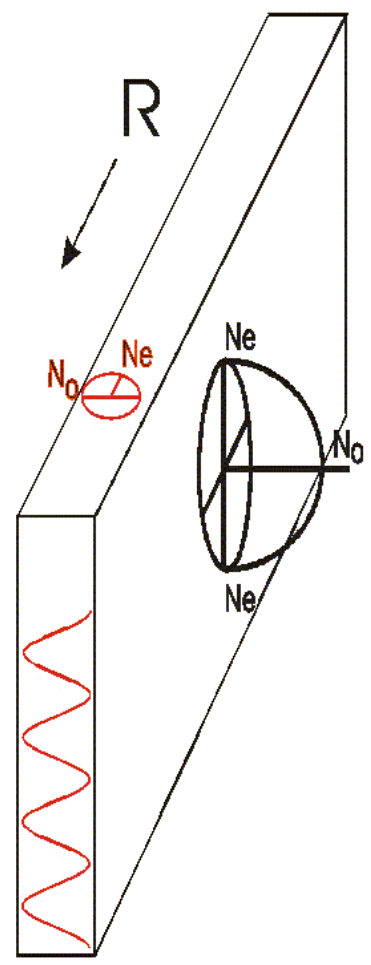

The lamellae in the hexagonites, as described by Varga, are disposed parallel to the base-surface on which they grow. On the surface of these formations tilted lamellae can form, which are brighter [26]. Stacks of lamellae, which are helically twisted in the 
radial direction, can be observed in ovals. The birefringence of hedrites is weaker than that of the ovals. The hedritic core consists of several dominant lamellae, and others which fill the space among them [28]. Varga and co-workers called this core a precursor. In accordance with unpublished data [25] a crystal with extended chain can serve as such a precursor. The primary lamellae with folded chains are disposed perpendicular to it in a structure called 'shish-kebab'. This type of structure could be oriented perpendicular to the base-surface on which it grows (Fig. 15a). Additional primary lamellae from the free material can become associated with this basic crystal. This precursor - when observed from above - seems like a hole (Fig. 15b), but actually it is a shish-kebab, which can incorporate different numbers of lamellae and have, respectively, different thickness, on the order of microns. The different images (Fig. 15c) which are observed in the central area of hedritic structures could be explained by different angles that the inclined crystals with extended chains in the centre made with the base-surface. With further growth of this structure, the lamellae splay, curve and split in radial direction. Such hedritic structures are growing by a screw dislocation mechanism.

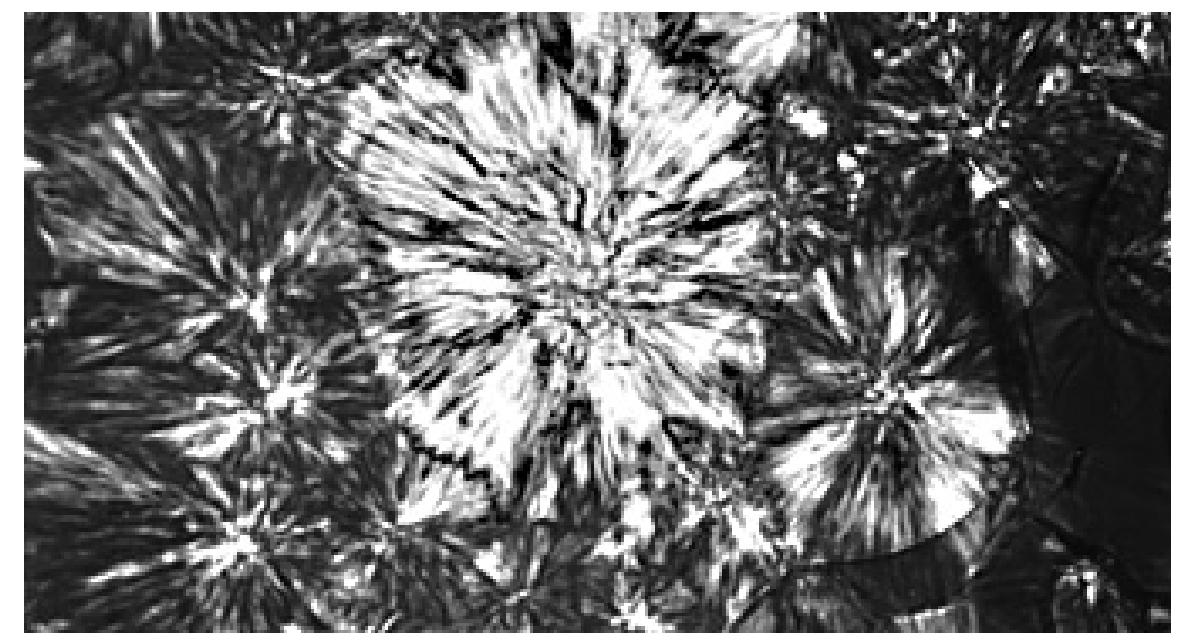

Fig. 14. Oval of the $\beta$ phase with approximately hexagonal form what can be determined by basic lamella; IPP, $200 \mathrm{x} ; T_{\mathrm{c}}=140^{\circ} \mathrm{C} ; t=17 \mathrm{~h}$

The $\beta$ spherulites form at crystallization temperature in the range $130^{\circ} \mathrm{C}<T<140^{\circ} \mathrm{C}$ (Fig. 16). The formation of a spherulite begins from a group of primary lamellae which are arranged similar to a sheaf. It has a hedritic core of the above described type (Fig. 15), from which the lamellae begin to grow out and form the circular boundary of the spherulites. Spherulites of the $\beta$ phase are built from lamellae growing straight radially (Fig. 16a,b) or from lamellae twisted along the radius (Fig. 16c).

The spherulites in Fig. 16b consist of edge-on lamellae, disposed radially (Fig. 13). They have strongly negative birefringence. Then we observe negative radial $\beta$ spherulites (Fig. 16b). When the lamellae are twisted along the radius, then banded negative spherulites form (Fig. 16c). This type of lamellae can be helically twisted along the $a$ axis which is the preferred fast growth direction $[11,26,28]$.

The birefringence of the banded spherulites is depicted in Fig. 17. The long axis of the polarizability ellipsoid at different points along the lengths of a twisted lamella is shown (Fig. 17). The resulting average optical axes of the twisting lamellae will incline at $45^{\circ}$ towards the axis of the incident beam [25] (Fig. 17). 


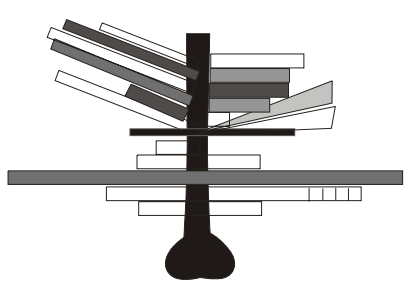

a.

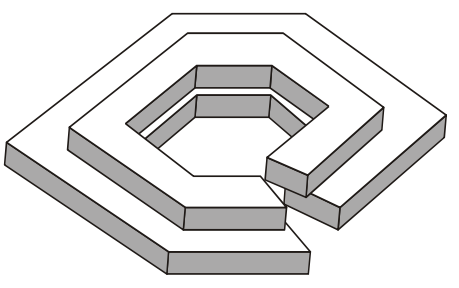

b.

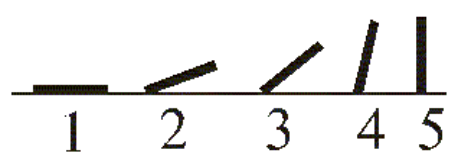

C.

Fig. 15. A nucleus of the precursor corresponding to the 'shish-kebab' type: (a) side view, (b) view from above, (c) different views to the crystal with extended chain (shish) towards the bottom over which it grows. The types of hedritic and oval centres, observed by Varga [11], could be explained this way

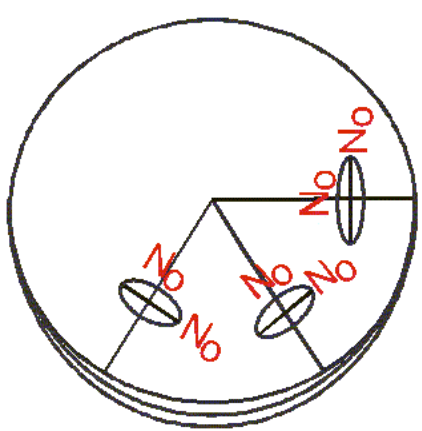

(a)

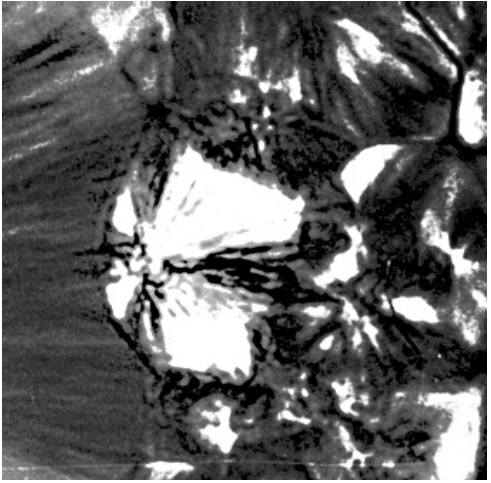

(b)

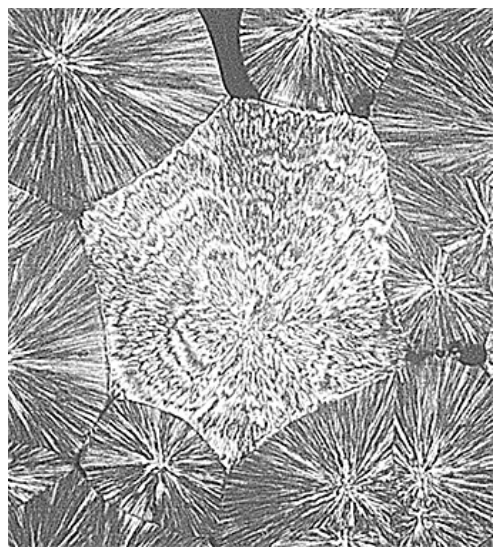

(c)

Fig. 16. (a) Negative $\beta$ spherulite. (b) Negative $\beta$ radial spherulite; IPP, 200x, with gypsum plate; $T_{\mathrm{c}}<140^{\circ} \mathrm{C}$ at non-isothermal conditions. (c) Negative $\beta$-banded spherulites; IPP, $200 \mathrm{x}, 90^{\circ}$ polarizers. $T_{\mathrm{C}}<140^{\circ} \mathrm{C}$ at non-isothermal conditions
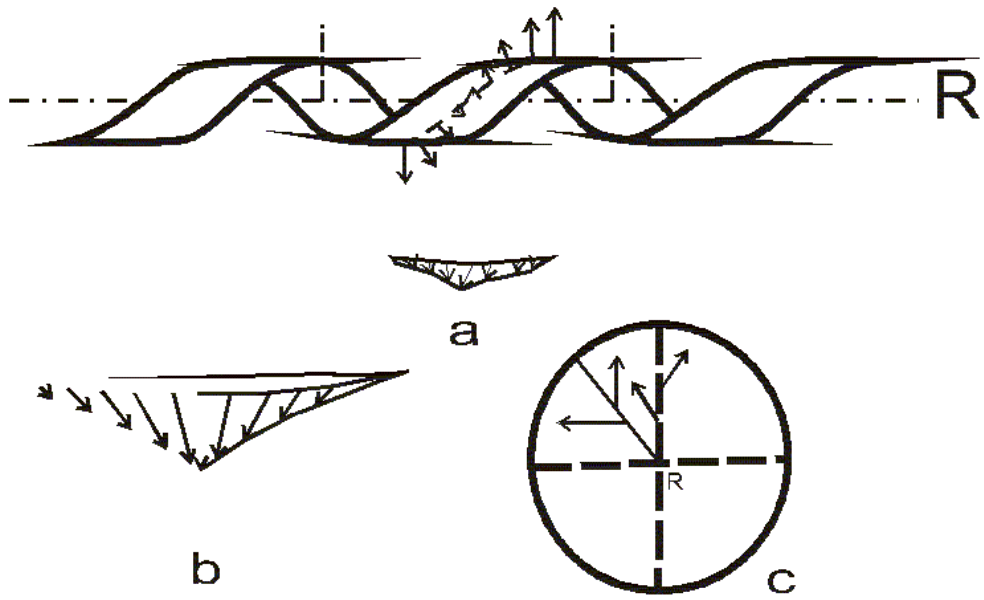

Fig. 17. Scheme of radial twisted lamellae in a banded spherulite. The direction of $\mathrm{N}_{\circ}$ is shown on half turn of the twisted lamella; (a) projections of the long axis $\mathrm{N}_{\circ}$ on the plane over which the spherulite grows; (b) projections of the the long axis $\mathrm{N}_{\circ}$ of a half turn of the twisted lamella; (c) sum of the projections of the the long axis $N_{\circ}$ of a half turn of a twisted lamella lies at $45^{\circ}$ towards the spherulitic radius 
Morphology of the $\gamma$-triclinic modification in dependence on the temperature of crystallisation

The $\gamma$ phase has a triclinic lattice with $a=6.47 \AA ; b=10.71 \AA ; c=10.99 \AA, \alpha=90^{\circ}$; $\beta=100^{\circ}$ and $\gamma=103^{\circ}$ [1]. Two type of helices - left- and right-handed - take part in this lattice (Fig. 18). These helices interact among themselves, not by methyl groups but by means of their planes [1]. The $\alpha$ and $\gamma$ forms have many narrow connections and, in fact, the $\gamma$ phase cannot occur without the $\alpha$ phase; in the first place since $\gamma$ crystals appear after the appearance of $\alpha$ crystals [8], and in the second place because both phases show very similar macromolecular packing [9]. The evidence for this is the easy transition of one phase to the other with a small change of crystallization conditions, only by a slip on the (100) plane $[8,9]$.

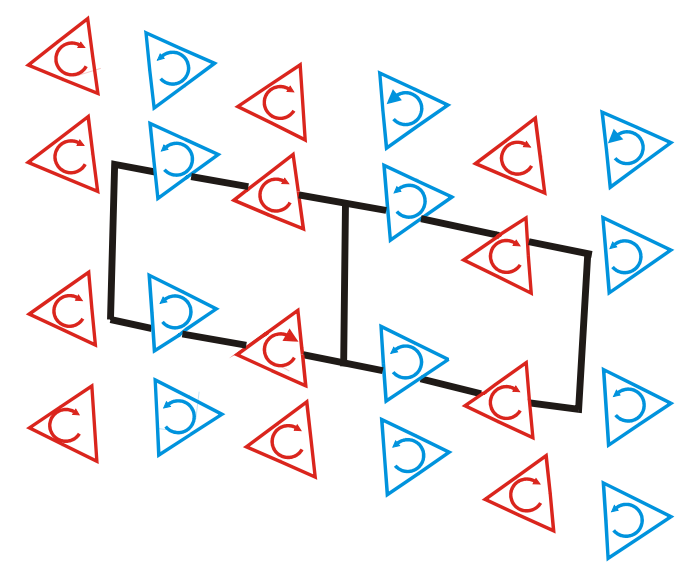

Fig. 18. (from ref. [1]) Basic $c$ projection of the $\gamma$ form of the lattice, with possible packing of left- and right-handed helices

$\gamma$ Lamellae form from extended, short chains $[8,9,15]$ which have a length equal to lamellar thickness. It is a proven fact that the thickness of $\gamma$ lamellae is smaller than that of $\alpha$ lamellae. The correlation of lamellar thickness is $\alpha: \gamma=1: 0,77$. $\alpha$ Lamellae consist only of folded chains while the preferred chains of the $\gamma$ lamellae are shorter and extended [9].

A $\gamma$ phase forms with low molecular weight IPP, for high molecular weight IPP at high pressure, and in irradiated IPP $[3,8,9,15]$. The $\gamma$ phase always appears with a nucleus of the $\alpha$ phase [8]. The $\gamma$ lamellae are elongated always along the $b^{*}$ axis. The difference between $\alpha$ and $\gamma$ phases, according to Lotz et al., is the preferable direction of fast growth. In $\alpha$ lamellae it is the a axis, in $\gamma$ lamella the $b^{*}$ direction. The helical chain axis of $\gamma$ lamellae is tilted always at $50^{\circ}$ towards the lamellar surface, whereas for $\alpha$ lamellae it is at $90^{\circ} . \gamma$ Lamellae can grow by a screw dislocation mechanism, but $\alpha$ lamellae cannot.

We obtained the mutual disposition of the optical and crystallographic axes in the $\gamma$ phase by analogy with the $\alpha$ phase. From basic crystallography it is known that the optical indicatrix is a non-rotational triaxial ellipsoid. Also it is not obligatory that any optical axis coincides with any crystallographic axis. By past observations [15] we know that supermolecular structures, consisting only of $\gamma$ lamellae, cannot obtained from the melt. Therefore, direct conoscopic observation and estimation of birefringence by means of a compensator is impossible. There are no data for these 
patterns and values in the literature, too. By means of conoscopic observations of a large group of $\gamma$ lamellae with size $<50 \mu \mathrm{m}$ and thickness $<30 \mu \mathrm{m}$, we determined only the disposition of the optical axes, i.e. the sign, for $\gamma$ lamellae. It is positive for the monoclinic phase. Consequently, $N_{p}$ is directed to the chain's axis. Our suggestion about the disposition of the optical indicatrix in relation to the lamellar surface (Fig. 20) is proved via birefringence determination of the more complicated supermolecular structures consisting of $\alpha$ and $\gamma$ branches, as spherulites and dendritic spherulites.

The unit cells of the $\alpha$ and $\gamma$ phases are approximately identical. The mutual situation of the axes of the optical indicatrix and the elementary cell of $\gamma$ lamellae is presented in Fig. 19. $N_{p}$ coincides with the $c$ axis. $N_{g}$ coincides with the $b$ axis, and $N_{m}$ is inclined to the a axis.

Fig. 19. Mutual disposition of crystallographic axes $a, b, c$ of the $\gamma$ crystal lattice with the axes of the polarizability ellipsoid, $\mathrm{N}_{\mathrm{m}}, \mathrm{N}_{\mathrm{p}}, \mathrm{N}_{\mathrm{g}}$. The sizes and angles of the crystal lattice are noted. The crystallographic axis $b$ coincides with $\mathrm{N}_{g}$
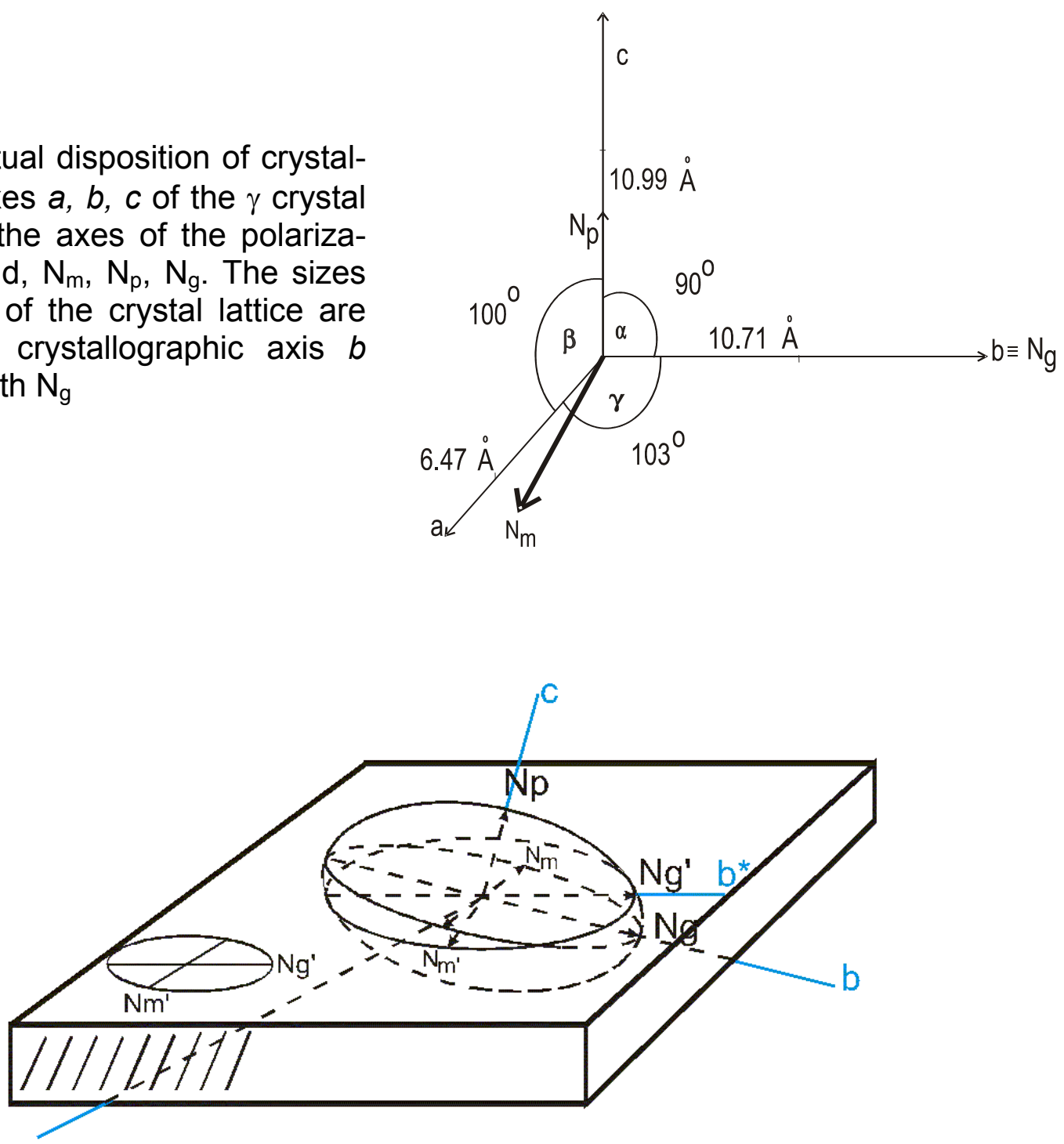

Fig. 20. $\gamma$ Lamella and its optical indicatrix; the a axis nearly coincides with $\mathrm{N}_{\mathrm{m}}, b^{*}$ coincides with $\mathrm{N}_{\mathrm{g}}$, and the $c$ axis coincides with $\mathrm{N}_{\mathrm{p}}$. The growth direction is the $b^{*}$ axis. The lamella is built by tilted, extended chains. The optical properties of this type of lamellae are determined by projections of the axes of the polarizability ellipsoid, $\mathrm{N}_{\mathrm{g}}$ ' and $\mathrm{N}_{\mathrm{m}}{ }^{\prime}$ 
The disposition of the crystallographic axes and the basic axes of the optical indicatrix to a triclinic $\gamma$ lamellae are shown in Fig. 20. The polarizability ellipsoid is rotated towards the lamellar surface because the $c$ axis is inclined to the lamella at an angle of $50^{\circ}$. The projection of the $b$ axis on the lamellar surface is $b^{*}$. The projections of $\mathrm{N}_{\mathrm{m}}$ and $\mathrm{N}_{\mathrm{g}}$ on the lamellar surface are $\mathrm{N}_{\mathrm{m}}$ ' and $\mathrm{N}_{\mathrm{g}}$ ', respectively. There is a great probability that one of the optical axes of the biaxial ellipsoid of a $\gamma$ lamella lies in the plane of the lamellar surface. But now we have no proofs of this and because of that we do not show them in Fig. 20.

The hedritic structure of $\gamma$ lamellae can grow by a screw dislocation mechanism [figure 5 in ref. 8] or epitaxially, and by branching from solution and melt [8,9]. It is very probable that the structure presented by Lotz et al. [figure 4 in ref. 8] is of the 'shish-kebab' type. This shish-kebab would serve as a precursor of a big flat-on lamellar structure with axial symmetry, i.e., a hedrite. Such hedrites have small sizes and can be observed only by transmission electronic microscopy. A detailed description of branching in the $\gamma$ phase was given by Lotz et al. [9] in their investigation of the quadrite type of the hedritic structure. The possible $\alpha-\alpha$ as well $\alpha-\gamma$ branchings are shown in Fig. 21 [15]. The latter always grow at $40^{\circ}$ towards the lamellar surface. The $b$ axis of the $\alpha$ phase remains parallel to the $b^{*}$ axis of the $\gamma$ phase [8].

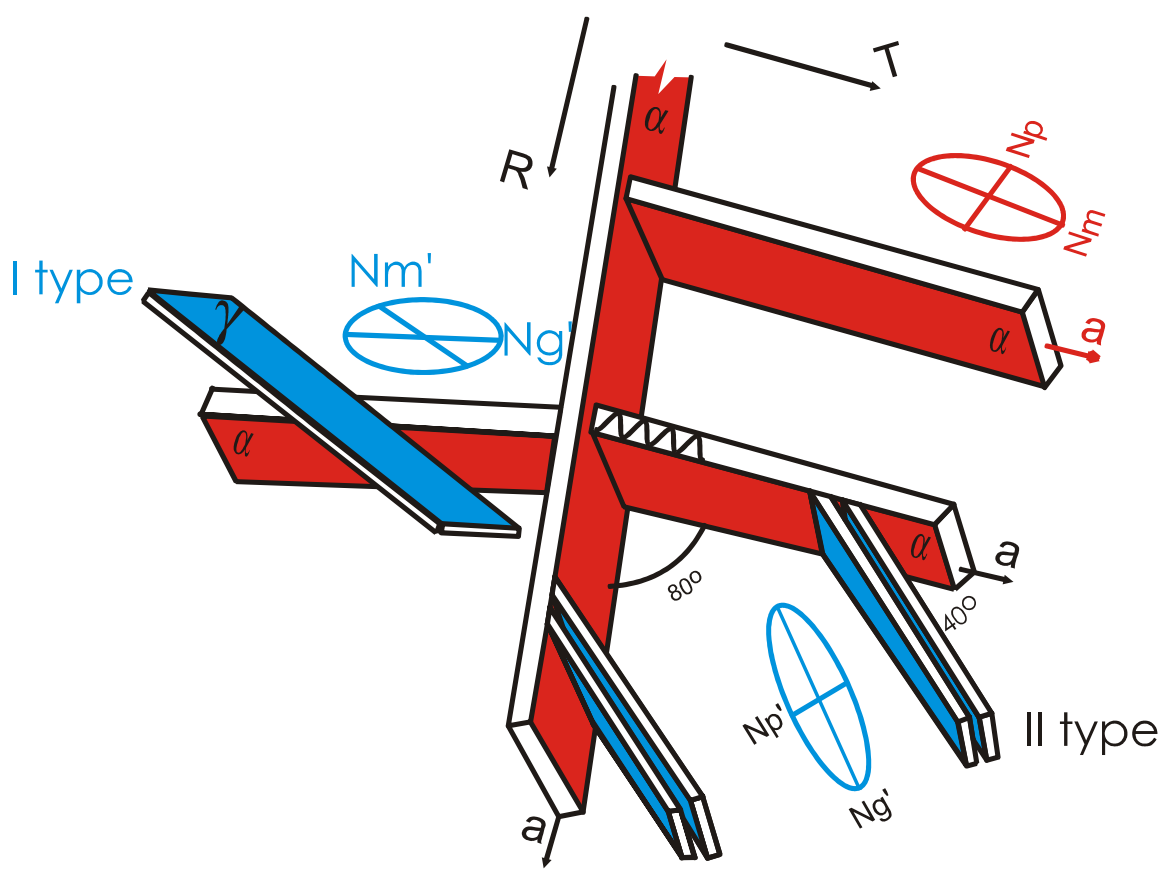

Fig. 21. (from ref. [15]) Formation of $\alpha-\alpha$ and two types of $\alpha-\gamma$ branches [15]. The observed birefringence is a result of the superposition of all types of polarizability ellipsoids, drawn in the figure. If the number of the two types $\alpha-\gamma$ branches increases than the magnitude of positive birefringence will increase

$\gamma$ Branchings are tilted and stacked in clusters. The growth of $\gamma$ phase lamellar clusters from type I and II (Fig. 21) can proceed by a screw dislocation mechanism. A work of Lotz et al. confirms the branching morphology in the quadrite, with the exception of considerably less tilt and larger flat-on $\gamma$ lamellae [9]. They were found to be in contact with the substrate over which the whole quadrite grows, but the contact 
with the parent $\alpha$ lamellae is on the edge. During their growth, $\gamma$ lamellae could topple over and continue to grow parallel to the substrate in the quadrite, as well as turn away at $40^{\circ}$ around the $c$ axis [9]. This method of growth, as well as the small sizes of the hedritic structures, makes the investigation of their optical properties difficult.

An investigation of sub-molecular structures of $\gamma$-ray irradiated IPP showed $\gamma-\gamma$ branching. As a result of irradiation, the bonds break easily. The chains are very short, probably extended chains, which easily form branched twins and polylamellar structures [15]. The spherulites, which are obtained during crystallization of irradiated PP with high doses of $\gamma$-rays, are strongly branched dendritics. The parent $\alpha$ lamellae are radially oriented, but the $\gamma$ lamellae are disposed at $40^{\circ}$ towards the parent one. $\mathrm{A}$ single spherulitic $\gamma$ lamella, with its optical indicatrix, is shown in Fig. 22. The birefringence is determined by the axes $\mathrm{N}_{\mathrm{g}}{ }^{\prime}$ and $\mathrm{N}_{\mathrm{p}}{ }^{\prime}$. The birefringence of a $\gamma$ lamella (Fig. 22 ) is larger than that of an $\alpha$-lamella (Fig. 7). Therefore, the birefringence in the first case is defined by $\left(\mathrm{N}_{\mathrm{g}}{ }^{\prime}-\mathrm{N}_{\mathrm{p}}{ }^{\prime}\right)$ and in the second case by $\left(\mathrm{N}_{\mathrm{m}}-\mathrm{N}_{\mathrm{p}}\right)$. The concentration of $\gamma$ crystals increases proportionately with the dose of irradiation until about $1500 \mathrm{kGy}$ [15]. The number of long, linear and cross-linked molecules decreases with increasing dose of irradiation. The number of short, non-defined molecules increases at high temperature. With a decrease of crystallization temperature pronounced $\alpha-\gamma$ branching occurs. So the number of $\alpha-\gamma$ branches is increased and, respectively, the observed positive birefringence (Fig. 21) is also increased. Because of that the mixed $\alpha-\gamma$ spherulites possess a larger positive birefringence than the $\alpha$ sherulites alone (Fig. 23). At lower temperatures, $\gamma$ lamellae can be packed in thick clusters by a screw dislocation mechanism.

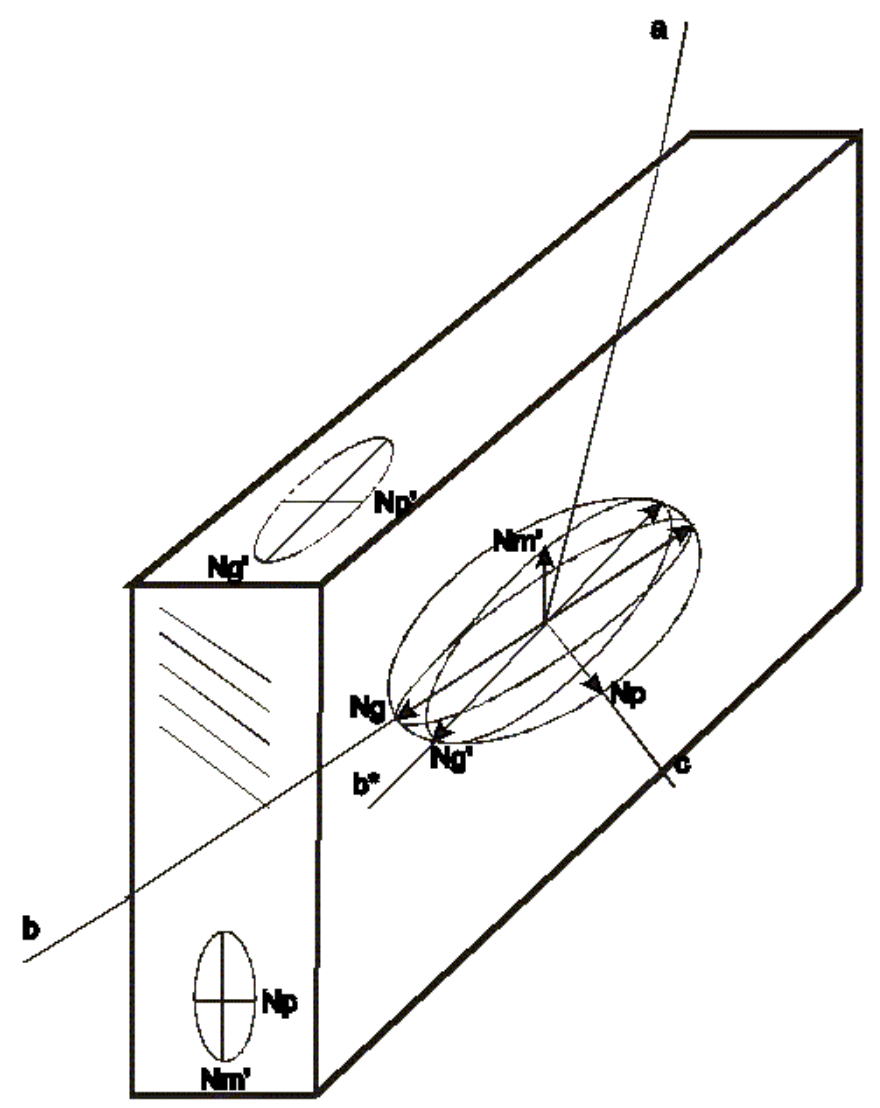

Fig. 22. The optical properties of a single spherulitic $\gamma$ lamella. This twin lamella grows at $40^{\circ}$ towards the basic $\alpha$ lamella 


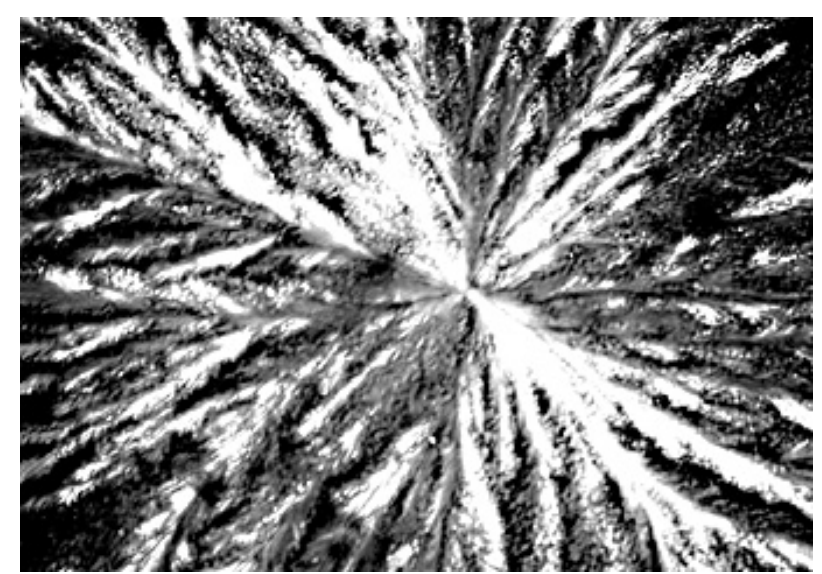

Fig. 23. A mixed spherulite with $\gamma$ - clusters. Irradiated IPP, $163 \mathrm{kGy} ; T_{\mathrm{c}}=120^{\circ} \mathrm{C}$ for $3 \mathrm{~h} ; 135 \mathrm{x}$, with gypsum plate

\section{Conclusion}

Under different crystallization conditions, the growth of different types of lamellae is observed, flat-on or edge-on, which can twist. Thus the supermolecular structures can have different birefringence - positive, zero and negative.

For the $\alpha$ phase the orientation of the axes of the polarizability ellipsoid is determined relative to the orientation of the unit cell. The axis $\mathrm{N}_{\mathrm{m}}$ coincides with the a axis, which is the direction of fastest growth. Hedritic structures grown by branching homoepitaxy show negative birefringence. The spherulites show variations in intensity and sign of birefringence because of cross-hatching.

For the $\beta$ phase the crystallographic $b$ axis coincides with $\mathrm{N}_{e}$, which is the short axis of the rotational polarizability ellipsoid. The hedritic structures can grow by the screw dislocation mechanism. The spherulites grow by an epitaxy mechanism. Both structures show negative birefringence.

The long axis $\mathrm{N}_{\mathrm{g}}$ of the polarizability ellipsoid of the $\gamma$ phase coincides with the $b^{*}$ axis. The short molecules of the $\gamma$ phase are inclined towards the lamellar surface at $40^{\circ}$. The unit cell axes and the axes of the polarizability ellipsoid are both inclined to the lamellar surface. $N_{g}$ coincides with the crystallographic axis $b^{*}$. Thus the positive birefringence of branching lamellae increases in the spherulites.

Acknowledgement: The authors would like to thank Prof. Dr. P. H. Geil for many useful discussions and morphological and style corrections.

[1] Turner Jones, J. A.; Aizelwood, J. M.; Beckett, D. R.; Makromol. Chem. 1964, 75, 134.

[2] Wunderlich, B.; Möller, M.; Grebowicz, J.; Baur, H.; Adv. Polym. Sci. 1984, 60, 57.

[3] Norton, D. R.; Keller, A.; Polymer 1985, 26, 704.

[4] Binsbergen, F. L.; DeLange, B. G. M.; Polymer 1968, 9, 23.

[5] Geil, P.H.; "Polymer single crystals", Ed. 'Chimija', Leningrad 1968, pp. $172 \mathrm{ff}$.

[6] Olley, R. M.; Hodge, A. M.; Basset, D. C.; J. Polym. Sci., Polym. Phys. Ed. 1979, 17,627 . 
[7] Lotz, B.; Wittmann, J. C.; J. Polym. Sci., Polym. Phys. 1986, 24, 1541.

[8] Lotz, B.; Graff, S.; Wittmann, J. C.; J. Polym Sci., Polym. Phys. 1986, 24, 2017.

[9] Lotz, B.; Graff, S; Straupe, Ch.; Wittmann, J. C.; Polymer 1991, 32, 2902.

[10] Ranby, B. G.; Morehead, F. F.; Walter, N. M.; J. Polym. Sci. 1960, 144, 349.

[11] Varga, J.; Ehrenstein, G. W.; Colloid Polym. Sci. 1997, 275, 511.

[12] Alamo, R. G.; Brown, G. M; Mandelkern, L.; Lehtinen, A.; Paukkeri, R.; Polymer 1999, 40, 3933.

[13] Chang-Mou Wu; Ming Chen; Karger-Kocsis, J.; Polymer 1999, 40, 4195.

[14] Nedkov, E.; Stoyanov, A.; Krestev, V.; Radiat. Phys. Chem. 1991, 37, 299.

[15] Nedkov, E.; Krestev, V.; Colloid Polym. Sci. 1990, 268, 1028.

[16] Varga, J.; "Structure and morphology" in "Polypropylene Structure, blends and composites", Karger-Kocsis , J., editor; Chapman and Hall, London 1995; pp. $57 \mathrm{ff}$.

[17] Varga, J.; Makromol. Chem. 1983, 112, 191.

[18] Kostov, I.; "Crystallography", Ed. 'Nauka i izkustvo', Sofia 1958, pp. $368 f f$.

[19] Atanasov, G.; Stojnov , H.; Vergilov , V.; "Practical guidance of petrography", Ed. 'Nauka i izkustvo', Sofia 1962, pp. $2 \mathrm{ff}$.

[20] Atanasov, A.; Nedkov, E.; Commun. Dept. Chem. Bulg. Acad. Sci. 1983, 16, 527, 541, 1984, 17, 131, 267.

[21] Michailov, M.; Nedkov, E.; Goshev, I.; J. Macromol. Sci., Part B 1978, 15, 313.

[22] Michailov, M.; Nedkov, E.; J. Polym. Sci., Part C 1972, 38, 33.

[23] Ferro, D.; Meille, S.; Brückner, S.; Macromolecules 1998, 31, 6926.

[24] Coulon, G.; Castelein, G.; G'Sell, C.; Polymer 1998, 40, 95.

[25] Nedkov, E.; Thesis, Bulg. Acad. Sci., 1974.

[26] Trifonova, D.; Varga, J.; Vancso, G. J.; Polym. Bull. (Berlin) 1998, 41, 341.

[27] Vancso, G. J.; Beekmans, L. G. M.; Pearce, R.; Trifonova, D.; Varga, J.; J. Macromol. Sci., Phys. 1999, 38, 491.

[28] Trifonova-van Haeringen, D.; Varga, J.; Ehrenstein, G. W.; Vancso, G.; J. Polym. Sci., Part B 2000, 38, 672. 